\title{
Problemas en la reparación por laser cladding de superficies de acero AISI D2 tratado térmicamente ${ }^{(\cdot)}$
}

\author{
J. J. Candel*, V. Amigó*, J. A. Ramos** y D. Busquets*
}

Resumen

Se ha depositado polvo de acero de herramienta (H13) sobre diferentes sustratos de acero que se encuentran en estado metalúrgico diferente (recocido o de temple y revenido) para comparar los resultados y se ha analizado la microestructura del recubrimiento y de la zona afectada por el calor (ZAC) mediante microscopia óptica, microscopía electrónica de barrido (MEB) y difracción de electrones retrodispersados (EBSD). El objetivo del trabajo es relacionar los parámetros del proceso de recubrimiento por láser (potencia, velocidad, caudal de polvo) con las transformaciones metalúrgicas producidas en el acero de herramienta AISI D2, con el fin de optimizar las condiciones de operación durante la reparación. Los resultados muestran que los parámetros influyen en gran medida en la geometría del cordón y en la formación de la microestructura. Aunque es sencillo obtener deposiciones geométricamente aceptables (forma y tamaño adecuado, ausencia de porosidad), en muchos casos aparecen fenómenos perjudiciales como la dilución de carburos y la aparición de fases metaestables que modifican las propiedades mecánicas del recubrimiento. En concreto, la presencia de austenita retenida en el recubrimiento, relacionada con la temperatura máxima alcanzada y la velocidad de enfriamiento, produce una fuerte disminución en la dureza, que debe evitarse. Además, para una misma composición y acabado superficial, el estado metalúrgico inicial del sustrato tiene una gran influencia sobre el resultado. Sustratos en estado de temple implican una mayor absorción del láser y la acumulación de calor que produce mayores zonas afectadas por el calor (ZAC). Por ese motivo, deben optimizarse los parámetros del proceso para cada operación de manera que se mejore el comportamiento en servicio del componente.

Palabras clave Recubrimiento láser; Acero de herramienta; EBSD; Austenita; Microestructura.

\section{Problems in laser repair-cladding a surface AISI D2 heat treated tool steel}

Abstract

The aim of the present work is to establish the relationship between laser cladding process parameters (Power, Process Speed and Powder feed rate) and AISI D2 tool steel metallurgical transformations, with the objective of optimizing the processing conditions during real reparation. It has been deposited $\mathrm{H} 13$ tool steel powder on some steel substrates with different initial metallurgical status (annealed or tempered) using a coaxial laser cladding system. The microstructure of the laser clad layer and substrate heat affected zone (HAZ) was characterized by Optical microscopy, Scanning Electron Microscopy (SEM) and Electron Backscattered Diffraction (EBSD). Results show that the process parameters (power, process speed, feed rate ...) determine the dimensions of the clad layer and are related to the microstructure formation. Although it is simple to obtain geometrically acceptable clads (with the right shape and dimensions) in many cases occur some harmful effects as carbide dilution and non-equilibrium phases formation which modify the mechanical properties of the coating. Specifically, the presence of retained austenite in the substrate-coating interface is directly related to the cooling rate and implies a hardness diminution that must be avoided. It has been checked that initial metallurgical state of the substrate has a big influence in the final result of the deposition. Tempered substrates imply higher laser absorption and heat accumulation than the ones in annealed condition. This produces a bigger HAZ. For this reason, it is necessary to optimize process conditions for each reparation in order to improve the working behaviour of the component.

Keywords Laser cladding; Tool steel; EBSD; Austenite; Microstructure.

\footnotetext{
(•) Trabajo recibido el día 8 de septiembre de 2009 y aceptado en su forma final el día 8 de febrero de 2010 .

* Instituto de Tecnología de Materiales, Universidad Politécnica de Valencia, España.

** Instituto Tecnológico de Óptica, Color e Imagen (AIDO) - Paterna, España.vamigo@mcm.upv.es; juacanbo@doctor.upv.es; dbusquets@mcm.upv.es; jramos@aido.es.
} 


\section{INTRODUCCIÓN}

El sector industrial del utillaje metálico (matricería, moldes y herramientas) es un sector altamente competitivo que requiere de técnicas que permitan aumentar la vida de las herramientas mediante su reparación y/o el aumento de sus prestaciones.

Este hecho es especialmente importante para el sector del molde ya que estos componentes tienen un coste muy elevado, trabajan en condiciones muy exigentes, requieren frecuentes modificaciones y su acabado tiene una importancia vital en el producto fabricado.

Por todos estos motivos, se han desarrollado numerosas técnicas de reparación y/o mejora de las prestaciones basadas en el recubrimiento de zonas concretas del componente.

Tradicionalmente, la reparación se llevaba a cabo mediante recargue por soldadura manual mediante una fuente de calor (por arco eléctrico por ejemplo) y añadiendo un hilo de aporte al baño fundido para recubrir el material dañado. En concreto, la soldadura por arco eléctrico con electrodo no consumible (TIG) ha sido ampliamente usada para esta aplica-

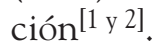

Sin embargo, un nuevo procedimiento de recubrimiento se ha ido desarrollando en los últimos años. Está basado en el uso del láser como fuente calorífica y el aporte de polvo metálico para formar un recubrimiento sobre la zona a modificar. La técnica concreta se denomina "laser cladding" (LC) o recubrimiento por láser. Desde el punto de vista práctico, el LC posee una gran cantidad de ventajas basadas en tiempos menores de operación, facilidad de automatización, mayor precisión y menor afectación térmica de la pieza recubierta ${ }^{[2-4]}$.

No obstante, desde el punto de vista metalúrgico, esta técnica es poco conocida en la reparación de algunos aceros para herramienta y se han producido diversos resultados contradictorios en cuanto al comportamiento en servicio del componente reparado ${ }^{[5]}$.

El calentamiento superficial por láser implica un aporte de energía sobre una zona muy localizada, de manera que se pueden alcanzar temperaturas superiores a $1.500 \mathrm{~K}$, seguido de un rápido enfriamiento de la zona afectada, en segundos ${ }^{[6]}$. Una correcta selección de los parámetros de procesamiento puede mejorar las propiedades superficiales del material pero, en caso contrario, no solo no se mejoran las propiedades sino que, incluso, puede favorecer el fallo prematuro del componente.

De la misma manera que ocurre con las fundiciones con alto contenido en cromo ${ }^{[7]}$, en los aceros para herramienta con alto contenido en cromo, un tratamiento térmico inadecuado produce la retención de austenita durante el enfriamiento, así como la inhibición de la precipitación de carburos. Este problema es especialmente importante durante el procesado láser de estos materiales y está fuertemente ligado a los parámetros del proceso ${ }^{[5-12]}$.

El tratamiento térmico de revenido, posterior a la reparación, puede ser una solución al problema ya que elimina la austenita retenida y favorece el endurecimiento por precipitación ${ }^{[7]}$. Sin embargo, no es una solución aceptable para muchos fabricantes de moldes que desean una reparación menor de un molde de gran tamaño.

Así pues, es importante determinar hasta qué punto es posible mejorar las propiedades del recubrimiento tan sólo con el control de los parámetros del proceso (potencia del láser, velocidad de avance y caudal de polvo aportado). Además, se analizará la defectología encontrada en las deposiciones realizadas y determinar las causas que originan dichos defectos.

\section{TRABAJO EXPERIMENTAL}

\subsection{Equipo láser empleado}

Los recubrimientos se han obtenido con un sistema láser de Nd:YAG, marca Trumpf modelo HL1006D de $1 \mathrm{KW}$ de potencia máxima operando en modo continuo. La propagación del haz láser se produce a través de una fibra óptica de $0,6 \mathrm{~mm}$ de diámetro en el núcleo. El sistema de focalización está compuesto por una lente con una focal de $200 \mathrm{~mm}$ que proporciona un diámetro del haz en el foco de 0,6 $\mathrm{mm}$.

La experimentación se ha llevado a cabo desenfocando el haz láser hasta obtener una haz de $2.5 \mathrm{~mm}$ aproximadamente, medido con un analizador de haz (Beam Monitor, Primes GMBH) siguiendo la norma UNE 11.146, para todas las potencias experimentadas. El perfil de irradiancia del haz a la distancia de desenfoque se corresponde a un perfil gaussiano.

Para depositar correctamente el polvo en el interior del baño fundido, así como proteger el recubrimiento de la oxidación, se ha empleado una boquilla coaxial con 3 orificios decalados $120^{\circ}$ y un orificio central por el que pasa el haz láser y el gas coaxial de protección. El gas empleado ha sido argón 5.0.

\subsection{Definición de materiales}

Como material base se ha empleado planchas metálicas de $5 \mathrm{~mm}$ de espesor, cortadas en plantillas de $100 \times 150 \mathrm{~mm}$. 
De entre todas las aleaciones férreas para trabajo en frío (menos de $200^{\circ} \mathrm{C}$ ) se ha elegido el acero EN 1.2379 (AISI D2) porque se ha detectado una problemática importante para su reparación mediante recubrimiento por láser. Se utilizaron planchas de acero en estado de suministro de recocido (a partir de ahora se indicará D2) y con un tratamiento térmico específico para cada una, de manera que se simule el proceso real de reparación (indicada como D2T). Estos aceros poseen una elevada dureza debido a la formación de carburos complejos de cromo y vanadio en una matriz de tipo martensítico, lo que permite obtener durezas por encima de $60 \mathrm{HRc}$ con el tratamiento térmico adecuado.

Para este trabajo se ha empleado un tratamiento de temple, calentando a $1.030^{\circ} \mathrm{C}$ para conseguir la disolución de los carburos, seguido de un enfriamiento en aceite. A continuación, se aplicó un revenido a $500{ }^{\circ} \mathrm{C}$ durante $2 \mathrm{~h}$ para eliminar tensiones y formar una segunda precipitación de carburos que aumenta la dureza útil. Para separar la influencia de las diferentes variables de estudio, también se ha depositado sobre acero al carbono $\mathrm{C} 45$ en dos estados de partida diferentes, en estado de recocido $(\mathrm{C} 45)$ y de temple en agua $(\mathrm{C} 45 \mathrm{~T})$.

Para mejorar los cordones, eliminar todos las imperfecciones, óxidos, grasas e igualar el acabado superficial de todos los sustratos empleados, las muestras se han cepillado y lijado con papel de $\mathrm{SiC}$ (grit 500) automático. Finalmente, se han desengrasado las superficies con acetona, en un baño de ultrasonidos.

Como material de aporte para realizar los recubrimientos se ha empleado polvo de acero de herramienta para trabajo en caliente EN 1.2344 (AISI H13) suministrado por TLS Technik ${ }^{\circledR}$ con una granulometría específica para aplicaciones láser y que está comprendida entre $30 \mu \mathrm{m}$ y $75 \mu \mathrm{m}$ con una morfología esférica. En la tabla I se recoge la composición de los sustratos y polvo empleados.

La elección de este material se debe a varios motivos. En primer lugar, debido a su endurecimiento por la formación de carburos complejos con molibdeno y/o vanadio, así como el contenido menor del cromo que permite obtener durezas entre 40-55HRc estables hasta una temperatura máxima de $800^{\circ} \mathrm{C}$ resultando más adecuado que el acero inoxidable AISI431, muy utilizado para la reparación de moldes. Además, el menor contenido en carbono que el del acero AISI D2 debería proporcionar al recubrimiento la tenacidad necesaria para evitar el comportamiento frágil y facilitar su templabilidad. De esta manera, se pretende evitar los problemas de formación de grietas y descenso de dureza. Finalmente, según las referencias encontradas, este material se emplea para la reparación de moldes y matrices de acero D2 mediante otras técnicas como soldadura TIG ${ }^{[13]}$.

\subsection{Recubrimientos obtenidos}

Existe un gran número de parámetros que pueden regularse en el proceso de LC. Sin embargo, no todos ellos tienen una influencia crítica en el proceso. A partir de la experiencia previa, se decide fijar diversos parámetros del proceso (como el caudal de gas de protección, la distancia al plano de trabajo o el diámetro del haz) en un valor que permite obtener recubrimientos correctos para el cabezal utilizado. Para evaluar el efecto de los parámetros más importantes, potencia, velocidad y caudal, se ha diseñado una tabla de experimentos. Estos parámetros y sus valores se recogen en la tabla II. En las plantillas fabricadas, para cada caudal de polvo, se ha dividido el rango de potencias en saltos de $100 \mathrm{~W}$ y el rango de velocidades en saltos de $0,1 \mathrm{~m} / \mathrm{min}$.

\subsection{Preparación metalográfica}

Se han cortado las plantillas y se ha preparado la sección transversal de cada cordón. Las muestras se han desbastado con papel de $\mathrm{SiC}$ y se han pulido con pasta de diamante hasta $0,25 \mu \mathrm{m}$ para observar la microestructura tanto del recubrimiento como del sustrato. Finalmente se ha atacado químicamente con

Tabla I. Composición química de los materiales utilizados como substrato y polvo de aporte (\% peso)

Table I. Chemical composition of powder and substrate material employed (\%wt)

\begin{tabular}{cccccccc}
\hline \% wt & $\mathbf{C}$ & $\mathbf{C r}$ & $\mathbf{S i}$ & Mo & $\mathbf{V}$ & $\mathbf{M n}$ & $\mathbf{F e}$ \\
\hline D2 & 1,55 & 11 & 0,3 & 0,75 & 0,75 & 0,3 & bal \\
H13 & 0,38 & 5,0 & 1,1 & 1,3 & 1,3 & 0,4 & bal \\
C45 & 0,45 & - & - & - & - & 0,71 & bal \\
\hline
\end{tabular}


Tabla II. Parámetros del procesado láser empleados para obtener los recubrimientos

\section{Table II. Processing parameters used for manufacturing tool steel coatings}

\begin{tabular}{lc}
\hline \multicolumn{1}{c}{ Parámetro de proceso } & Valor fijado \\
\hline Gas & Argón 5.0 \\
Caudal gas impulsor & $4 \mathrm{l} / \mathrm{min}$ \\
Caudal gas coaxial & $4 \mathrm{I} / \mathrm{min}$ \\
Diámetro haz & $2,5 \mathrm{~mm}$ \\
Potencia & $400-800 \mathrm{~W}$ \\
Caudal polvo & $6-14 \mathrm{~g} / \mathrm{min}$ \\
Velocidad & $0,5-1 \mathrm{~m} / \mathrm{min}$ \\
\hline
\end{tabular}

reactivo de Vilella (95 $\mathrm{ml}$ Etanol, $5 \mathrm{ml} \mathrm{HCl}, 1 \mathrm{~g}$ Ácido Pícrico).

\subsection{Técnicas de análisis empleadas}

Se ha analizado el corte transversal de los recubrimientos mediante microscopia óptica para identificar los posibles defectos, determinar las dimensiones del baño fundido y obtener la distribución de fases. En concreto, para el análisis geométrico se ha medido la altura y anchura del cordón y de la ZAC $(\mathrm{Hc}, \mathrm{Wc})$ y $\left(\mathrm{Hz}, \mathrm{Wz}_{\mathrm{z}}\right.$ ), respectivamente (Fig. 1).

A continuación, se han obtenido detalles a mayor resolución con ayuda de microscopía electrónica de barrido así como microanálisis mediante espectroscopía por energías dispersivas, para determinar la microestructura y la composición en cada una de las zonas de interés. El análisis microestructural se completa

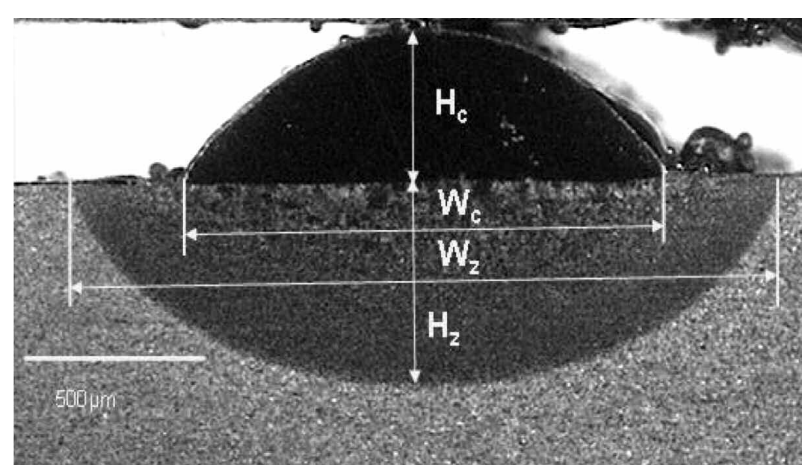

Figura 1. Parámetros geométricos medidos en uno de los recubrimientos sobre acero C45.

Figure 1. Clad geometrical parameters that have been measured in C45 substrate. mediante difracción por electrones retrodispersados. En concreto, se utilizará el mapa de distribución de fases para identificar y cuantificar las fases presentes a nivel local del recubrimiento. De esta manera, se puede relacionar las imágenes y análisis por EDS con las fases formadas durante el procesado.

Se completa la caracterización con barridos de microdureza Vickers desde el metal base hasta la superficie del cordón para evaluar la influencia de los cambios microestructurales sobre las propiedades. La carga empleada ha sido de $300 \mathrm{~g}$ aplicada durante $10 \mathrm{~s}$.

\section{RESULTADOS Y DISCUSIÓN}

\subsection{Análisis geométrico}

El primer paso para realizar una reparación consiste en determinar los parámetros qué deben emplearse para obtener un cordón con unas dimensiones adecuadas. En concreto, según Steen y Oliveira ${ }^{[8 \text { y 14] las }}$ dos propiedades geométricas más importantes a controlar son: la altura del cordón (Hc) y su relación de aspecto (RA) entendida como la relación entre la anchura y la altura del cordón. Cordones demasiado altos, tienden a acumular el calor en su interior. De esta manera se alcanzan temperaturas demasiado altas en el recubrimiento y favorecen la falta de adherencia. La relación de aspecto, está fuertemente relacionada con la aparición de porosidad y grietas en el solapamiento entre cordones. Por tanto, se recomienda utilizar cordones cuya relación de aspecto sea mayor que cinco. No debe olvidarse tampoco, la penetración del recubrimiento en el substrato ya que debe reducirse a la mínima expresión posible para disminuir la afectación térmica del molde o utillaje a reparar.

De acuerdo con la bibliografía ${ }^{[8}$ y 14], la altura del cordón está relacionada con el parámetro P/VD entendido como el cociente entre la potencia de láser (P), la velocidad de proceso (V) y el diámetro del haz (D); este parámetro se puede expresar en $\mathrm{J} / \mathrm{mm}^{2}$ y representa la densidad superficial de energía aportada. De la misma manera, se puede relacionar la relación de aspecto con el parámetro M/VD, siendo M el caudal de polvo soplado y que representa la cantidad de polvo soplado por unidad de superficie. Esta relación, RA vs. M/VD, se cumplirá siempre que se mantenga la potencia por encima de un umbral que provocaría recubrimientos discontinuos para un caudal de polvo demasiado alto.

Para la diversidad de materiales objeto de estudio, se ha encontrado que se produce un mejor ajuste si se emplea la medida de la anchura del cordón 
(Wc) en vez del diámetro teórico (D). Esto, puede ser debido a la gran diferencia estructural y las propiedades térmicas de los substratos de partida. El acero para herramienta y en particular en estado de temple, presenta una absorción mayor de la radiación láser que cualquiera de los otros materiales. Además, los aceros de alta aleación pueden sufrir fuertes variaciones en su calor específico $\mathrm{Cp}(\mathrm{T})$ durante el calentamiento que incrementa aún más la zona afectada por el calor ${ }^{[6]}$.

En la figura 2 se muestra la relación lineal encontrada entre la altura del cordón y la energía específica aportada (P/VD). Para un par recubrimiento-substrato en concreto y fijando el caudal de polvo, a mayor energía mayor altura (Fig. 2 A)). Es importante destacar cómo el coeficiente de ajuste de regresión lineal disminuye conforme aumenta el caudal. Este fenómeno está ligado a la variación de la eficiencia del proceso debido al apantallamiento del polvo, la modificación de la energía disponible para cada par-

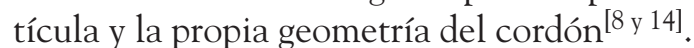

Si se fija el caudal y se compara el efecto del substrato se observa que el substrato de acero AISI D2 con tratamiento térmico previo produce una mayor altura del cordón, manteniendo todos los parámetros del proceso constantes, llegando incluso a doblar las dimensiones del mismo recubrimiento sobre acero al carbono (Fig. 2 B)). Esta mayor gran acumulación de calor en el cordón favorece una mayor penetración en el substrato, debido a una mayor densidad de energía efectiva sobre la intercara sólido-líquido ${ }^{[15]}$. En la tabla III se muestran los
Tabla III. Coeficientes de correlación $\mathrm{R}^{2}$ para la altura del baño fundido vs P/VD

Table III. $R^{2}$ correlation coefficients for the height of the melt vs. PND

\begin{tabular}{cllll} 
Caudal (g/min) & C45 & C45T & D2 & D2T \\
\hline 14 & 0,9285 & & & \\
10 & 0,9563 & & & \\
8 & 0,9611 & & & \\
6 & 0,9727 & 0,8492 & 0,908 & 0,9218
\end{tabular}

coeficientes de correlación encontrados para las diferentes condiciones.

La relación de aspecto también está fuertemente afectada por el substrato. En la figura 3, se representa (RA) frente a M/VD. Efectivamente, si la potencia es suficiente para obtener un recubrimiento continuo, no afecta de manera importante a la relación de aspecto. Como puede observarse, cuando se emplea polvo de acero para herramienta, es bastante complicado obtener recubrimientos con RA mayor que cinco, pero cuando el substrato es acero AISI D2 templado es, prácticamente, imposible. Otra diferencia remarcable es que para el acero al carbono C45, prácticamente, no hay diferencia en el RA si el substrato esta tratado térmicamente o no, sin
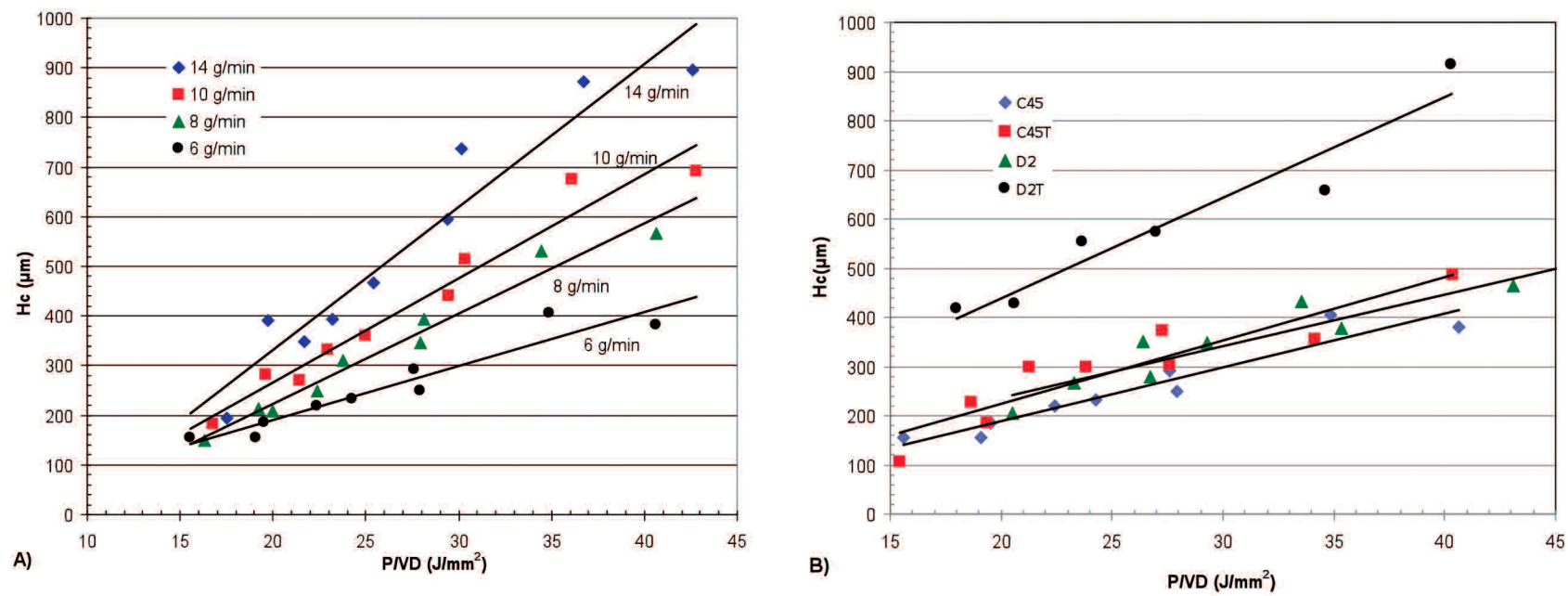

Figura 2. Altura del cordón en función de la energía aportada (P/VD) para diferentes caudales de polvo. A) Dependencia de Hc con la variación caudal de polvo sobre la altura del recubrimiento para substrato C45. B) Efecto del substrato utilizado sobre la altura, para un caudal de polvo fijo de $6 \mathrm{~g} / \mathrm{min}$.

Figure 2. Laser clad height (Hc) dependence on the specific energy and feeding rate parameters. A) $\mathrm{Hc}$ as a function of powder feeding rate and specific energy for fixed C45 substrate; B) Hc as function of specific energy and substrate combination. 


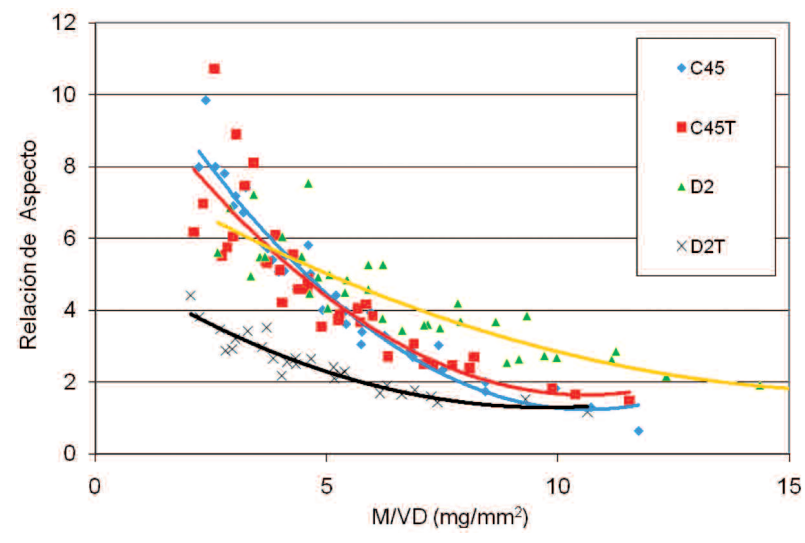

Figura 3. Efecto de la densidad de polvo aportado sobre la relación de aspecto del cordón.

Figure 3. Effect of powder density on single track Aspect Ratio.

embargo, la diferencia es clara para el acero para herramienta. Esta diferencia se aclarará mediante el análisis microestructural.

\subsection{Caracterización del substrato}

En la figura 4 se observa la estructura del acero D2 en estado de suministro y el comienzo de la ZAC debajo del recubrimiento. En estado de recocido, los carburos de cromo se encuentran en forma de esferoides y partículas de gran tamaño, en una matriz ferrítica. Cuando se deposita el recubrimiento, la zona superior del substrato y más próxima al cordón

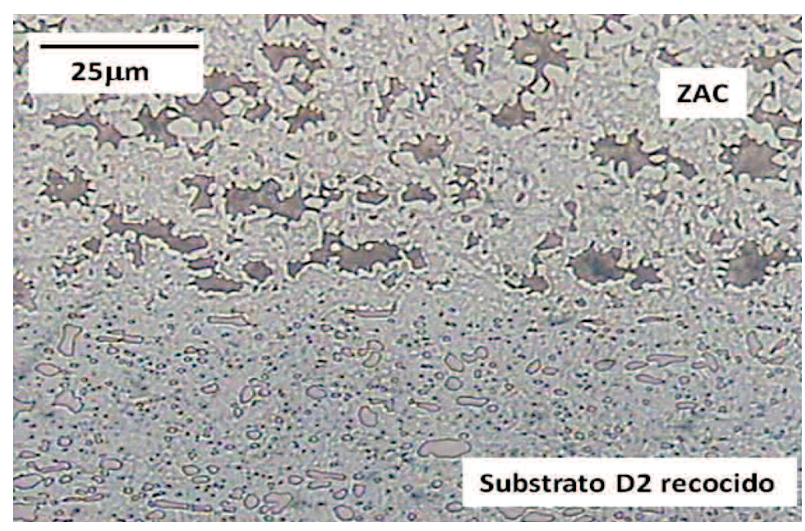

Figura 4. Frontera entre el substrato de acero AISI D2 y la Zona Afectada por el Calor durante el procesado. Cordón con energía $\mathrm{E}=30 \mathrm{~J} / \mathrm{mm}^{2}$

Figure 4. Interface between the Heat Affected Zone and AISI D2 substrate. se ve fuertemente afectada por el calor, los carburos se diluyen y aparece la transformación austenítica del acero ${ }^{[6]}$. Sin embargo, debido a la gran velocidad de enfriamiento, gran parte de la austenita queda retenida. La corta duración del ciclo térmico, la elevada temperatura alcanzada y la disminución de la solubilidad del carbono y de los elementos de aleación en la austenita durante su enfriamiento, favorecen que gran parte de los elementos de aleación permanezcan en borde de grano ${ }^{[7]}$.

Tras el tratamiento térmico, parte de los carburos se diluyen en la matriz y precipitan en forma de partículas más finas que endurecen la matriz martensítica formada durante el temple (Fig. 5 D)). Las imágenes obtenidas mediante electrones retrodispersados confirman que hay una fina dispersión de carburos cuya composición es diferente a la de la matriz de hierro. Además, se puede observar carburos de pequeño tamaño que han precipitado en el interior del grano austenítico previo. La identificación de fases en el substrato, mediante EBSD (Fig. 6 A)) muestra que la microestructura está formada, principalmente, por martensita (37\%), carburos complejos de cromo-hierro $(\mathrm{Fe}, \mathrm{Cr})_{23} \mathrm{C}_{6}(40 \%) \mathrm{y}$, tan solo, aparecen carburos de cromo puros $\mathrm{Cr}_{3} \mathrm{C}_{2}$ finamente precipitados en la matriz (7\%). Además, se ha detectado una pequeña cantidad de austenita retenida $(5 \%)$ y algunos carburos no se han conseguido disolver correctamente $^{[8,16 \text { y } 17] \text {. }}$

\subsection{Caracterización de los recubrimientos}

En todos los substratos empleados ha sido posible obtener cordones libres de defectos macroestructurales (grietas y/o poros) y la microestructura del cordón presenta la misma estructura (Fig. 7). Hay una primera piel a partir de la intercara, de pocas micras de espesor y cuya estructura no está claramente definida. A continuación, debido a la menor velocidad de enfriamiento, la microestructura evoluciona hacia una zona columnar, que termina con la formación de dendritas ramificadas. Finalmente, en el centro del cordón se encuentra una estructura bastante redondeada debido a que es el último líquido solidificado en el cordón. No obstante, la extensión de cada una de estas zonas es diferente según varíen los parámetros de proceso o el substrato. Sobre acero al carbono y con baja energía aportada, la primera zona que aparece a partir de la intercara es de mayor extensión y el tamaño de las dendritas es mucho menor. Pero si la energía aportada es alta o si el substrato presenta una conductividad menor, como es el caso del acero para herramientas, se forman grandes 

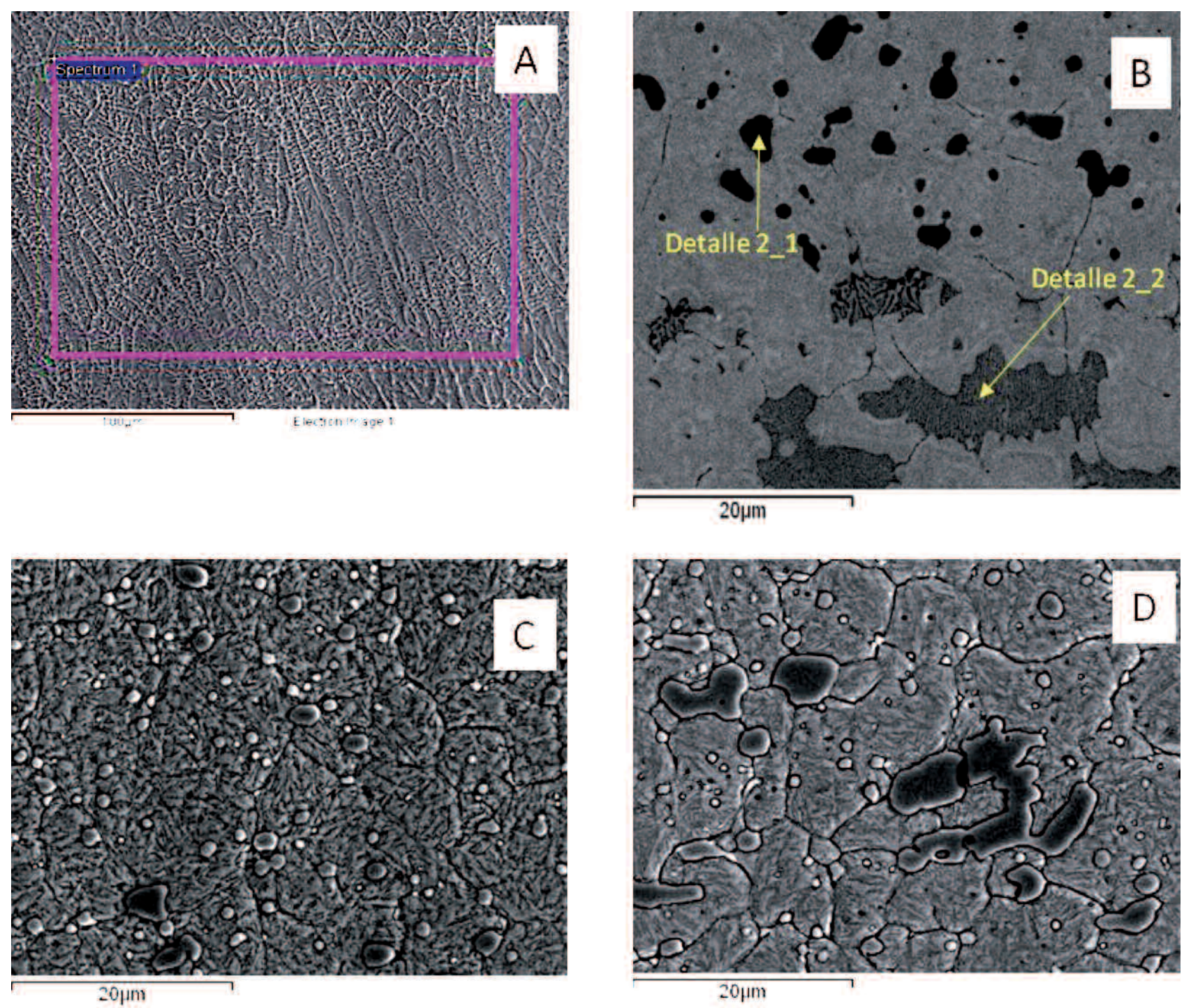

Figura 5. (a) Microestructura del cordón de acero H13. (b) Granos austeníticos presentes en la interfase substrato-cordón. (c)Microestructura de la zona afectada por el calor. (d) Microestructura del substrato de acero D2T.

Figure 5. (a) Microstructure of $\mathrm{H} 13$ tool steel clad layer. (b) Austenite grains presents at the interface between clad and substrate. (c) Heat Affected Zone microstructure. (d) Initial microstructure of the AISI D2 heat treated substrate.

dendritas de tipo columnar, sin brazos secundarios, por crecimiento epitaxial a partir del grano austenítico previo del substrato, (Fig. 5 A)). A medida que el gradiente térmico disminuye hacia el centro del cordón, el tamaño de las dendritas disminuye y llegan a formarse brazos secundarios. Ya, en el centro del cordón, donde se produce la última solidificación, aparecen granos de tipo celular ${ }^{[10-12}$ y 16-18].

Para el análisis por EDS y EBSD se han seleccionado dos condiciones diferentes, según la energía aportada de $\mathrm{E}=20 \mathrm{~J} / \mathrm{mm}^{2}(\mathrm{P}=600 \mathrm{~W} ; \mathrm{V}=1 \mathrm{~m} / \mathrm{min})$ y $30 \mathrm{~J} / \mathrm{mm}^{2}(\mathrm{P}=800 \mathrm{~W} ; \mathrm{V}=0,6 \mathrm{~m} / \mathrm{min})$.
Se ha comprobado mediante análisis por EDS que, debido a la difusión y al movimiento del material fundido dentro del cordón, existe una transferencia de carbono y elementos de aleación entre el substrato de acero AISI D2T y el recubrimiento. En el centro del cordón (espectro 1, Fig. 5 A)) la composición en peso registrada ha sido de: 0,81 \% C, 0,82 \% Si, 0,47 \% V, 5,57 \% Cr, 1,34 \% Mo y Fe bal. Por tanto, hay un enriquecimiento en carbono en el recubrimiento, acompañado de pérdida de silicio y vanadio. Estas variaciones son directamente proporcionales a la energía aportada durante el procesado láser (Tabla IV). 

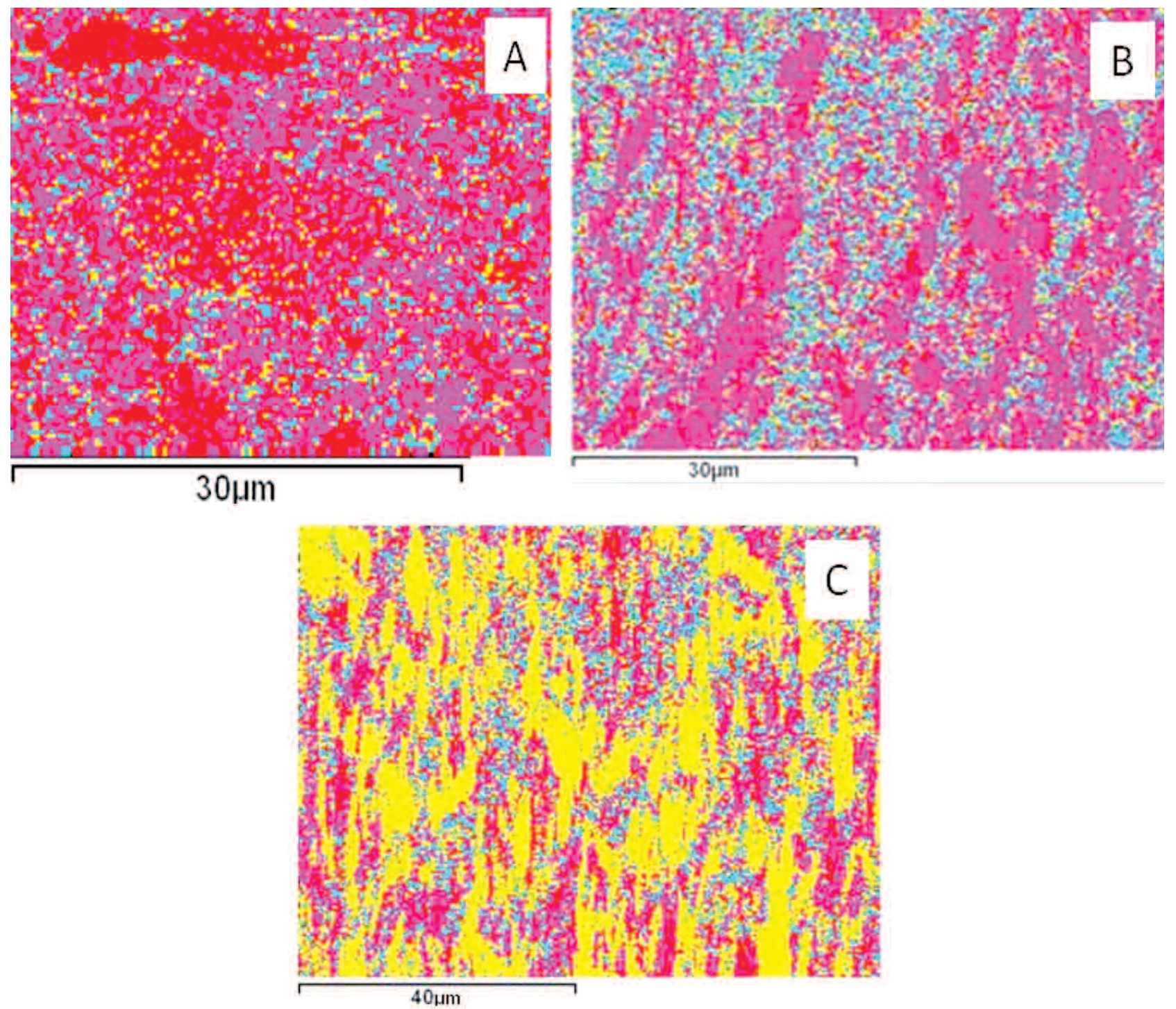

Figura 6. Análisis mediante EBSD del recubrimiento mostrado en la figura 5. A) Substrato D2T (detalle D); B) Cordón (detalle A); C) Interfase (detalle B). Amarillo = Austenita, Rosa = Martensita, Rojo $=(\mathrm{Fe}, \mathrm{Cr})_{23} \mathrm{C}_{6}, \mathrm{Azul}=\mathrm{Cr}_{3} \mathrm{C}_{2}$.

Figure 6. EBSD analysis of the clad showed in the figure 5. A) D2T substrate (detalle D); B) Clad $\left(\right.$ detalle A); C) Interface (detalle B). Yellow = Austenite, Pink $=$ Martensite, $\mathrm{Red}=(\mathrm{Fe}, \mathrm{Cr})_{23} \mathrm{C}_{6}$, Blue $=$ $\mathrm{Cr}_{3} \mathrm{C}_{2}$.

La zona afectada por el calor (ZAC) presenta una microestructura de tipo martensítico (Fig. 5 C)) y si se compara con la del substrato previo (Fig. 5 D)) se observa una tamaño menor de los carburos.

En la zona del substrato de acero D2T más cercana a la intercara con el recubrimiento se aprecia una microestructura de tipo austenítico (Fig. 5 B)). Debido a la gran temperatura alcanzada, se aprecia un crecimiento importante del grano ${ }^{[7]}$. Además, en la imagen en electrones retrodispersados, se observan, en color más oscuro, los elementos de aleación más ligeros que están en borde de grano. El análisis mediante EDS de los detalles mostrados en la figura $5 \mathrm{~B}$ ), se recoge en la tabla V. Efectivamente, los elementos de aleación son desplazados al borde de grano. Además, se puede apreciar una transición entre la microestructura de tipo eutéctico muy fino en la parte inferior (detalle 2_2) hasta una precipitación más rica en carburos en la parte superior (detalle 2_1). Este efecto es debido a la mayor temperatura alcanzada conforme se asciende hacia el recubrimiento y un mayor tiempo de enfriamiento ${ }^{[9-12]}$.

Con el análisis mediante EBSD, gracias al mapa de distribución de fases, se puede identificar y 


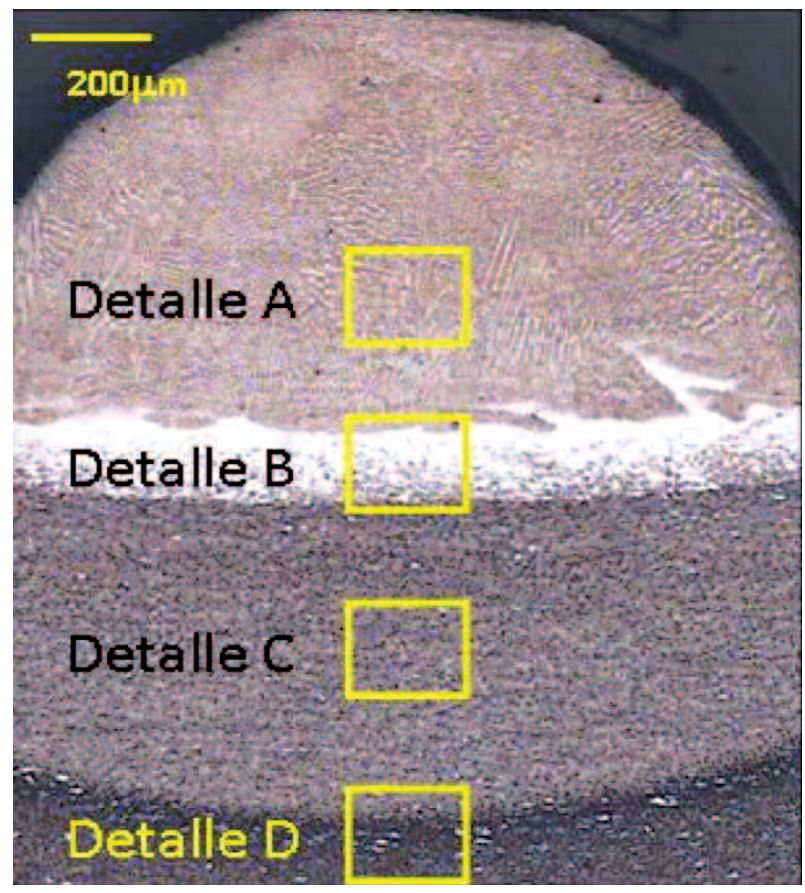

Figura 7. Corte transversal del recubrimiento con acero H13 sobre substrato de acero D2T. Cordón con $\mathrm{E}=20 \mathrm{~J} / \mathrm{mm}^{2}$.

Figure 7. Cross section of laser clad H13 steel layer deposited on D2T steel substrate.

cuantificar las fases presentes en la zona de dilución (Fig. 6 C)). Se confirma que hay gran cantidad de austenita retenida. Para una energía aportada de 30 $\mathrm{J} / \mathrm{mm}^{2}$ aparece un $43 \%$ de austenita y el resto de fases. en proporciones aproximadamente iguales $\left((\mathrm{Fe}, \mathrm{Cr})_{23} \mathrm{C}_{6} 17 \%\right.$, martensita $15 \%$ y $\mathrm{Cr}_{3} \mathrm{C}_{2} 11,5$ $\%)$. El análisis de la influencia de los parámetros muestra que la cantidad de austenita está fuertemente condicionada por la energía específica aportada (P/VD) que condiciona la velocidad de enfriamiento. A menor energía aportada, mayor cantidad de austenita retenida ${ }^{[9-12]}$. El análisis del cordón depositado con una energía de $20 \mathrm{~J} / \mathrm{mm}^{2}$ muestra un mayor contenido en austenita hasta el 57 \% (Tabla VI).
Tabla V. Análisis EDS de los detalles marcados en la figura 6B

Table V. EDS analysis of remarked details in figure $6 B$

\begin{tabular}{ccrcc}
\hline & \multicolumn{2}{c}{ Detalle 2_2 } & \multicolumn{2}{c}{ Detalle 2_1 } \\
\cline { 2 - 5 } Elemento & $\begin{array}{c}\text { \% } \\
\text { peso }\end{array}$ & $\begin{array}{c}\text { \% } \\
\text { atómico }\end{array}$ & $\begin{array}{c}\text { \% } \\
\text { \%peso }\end{array}$ & $\begin{array}{c}\% \\
\text { atómico }\end{array}$ \\
\hline $\mathrm{C}$ & 12,51 & 39,53 & 13,38 & 40,97 \\
$\mathrm{Cr}$ & 21,9 & 15,98 & 44,29 & 31,32 \\
$\mathrm{~V}$ & 2,09 & 1,56 & 4,48 & 3,23 \\
$\mathrm{Si}$ & 0,23 & 0,31 & & \\
$\mathrm{Fe}$ & 61,66 & 41,89 & 36 & 23,7 \\
$\mathrm{Mn}$ & 0,31 & 0,21 & & \\
$\mathrm{Mo}$ & 1,3 & 0,51 & 1,58 & 0,6 \\
\hline
\end{tabular}

La identificación de las fases presentes en la zona dendrítica del cordón muestra que el cuerpo principal de la dendrita está formado por martensita con una fina dispersión de carburos mixtos de hierro-cromo en su interior $y$, finalmente, los pequeños carburos de cromo $\left(\mathrm{Cr}_{3} \mathrm{C}_{2}\right)$ se encuentran en los espacios entre dendritas ${ }^{[18]}$. Al igual que en la intercara, puede aparecer austenita retenida si disminuye la energía aportada y aumenta la velocidad de enfriamiento. No obstante, debido al menor contenido en carbono y elementos de aleación del acero $\mathrm{H} 13$ no llega a superarse el $10 \%$ de austenita retenida en ninguno de los casos analizados. Por tanto, el polvo de acero H13 parece ser un buen material para reparar moldes de acero D2 ya que estos resultados no hubiera sido posible conseguirlos con el polvo de acero D2.

\subsection{Medida de la dureza}

Se han realizado barridos la dureza para las dos condiciones anteriormente mencionadas (Fig. 8). La

Tabla IV. Resumen de los análisis por EDS de la zona central de cada cordón. Se muestra el porcentaje en peso de cada elemento

Table IV. Summary of EDS analysis of the central zone of each track. It shows the percentage weight of each item

\begin{tabular}{ccccccc}
\hline Identificación & $\mathbf{C}$ & $\mathbf{S i}$ & $\mathbf{V}$ & $\mathbf{C r}$ & Mo & Fe \\
\hline $20 \mathrm{~J} / \mathrm{mm}^{2}$ & 0,81 & 0,82 & 0,47 & 5,57 & 1,34 & $\mathrm{bal}$ \\
$30 \mathrm{~J} / \mathrm{mm}^{2}$ & 1,29 & 0,74 & 0,43 & 6,61 & 1,26 & $\mathrm{bal}$ \\
\hline
\end{tabular}


PROBLEMAS EN LA REPARACIÓN POR LASER CLADDING DE SUPERFICIES DE ACERO AISI D2 TRATADO TÉRMICAMENTE PROBLEMS IN LASER REPAIR-CLADDING A SURFACE AISI D2 HEAT TREATED TOOL STEEL

Tabla VI. Resumen de los resultados de la cuantificación de fases en las diferentes zonas de interés

Table VI. Summary of the results of the quantification of phases in different areas of interest

\begin{tabular}{lccccc}
\hline \multicolumn{1}{c}{ Identificación } & No resuelto & $\mathbf{F e} \gamma$ & Fe martensita & $\mathbf{C r}_{\mathbf{3}} \mathbf{C}_{\mathbf{2}}$ & $\mathbf{M}_{\mathbf{2 3}} \mathbf{C}_{\mathbf{6}}$ \\
\hline Interfase 30J/mm² & 11,6 & 43 & 15,9 & 11,5 & 17 \\
Interfase 20J/mm & 10,2 & 57,1 & 11 & 11,6 & 10,2 \\
Sustrato D2T & 9,5 & 5,2 & 37 & 7,6 & 40,7 \\
Centro cordón 20J/mm & 21,3 & 8,7 & 33,6 & 16,2 & 20,2 \\
\hline
\end{tabular}

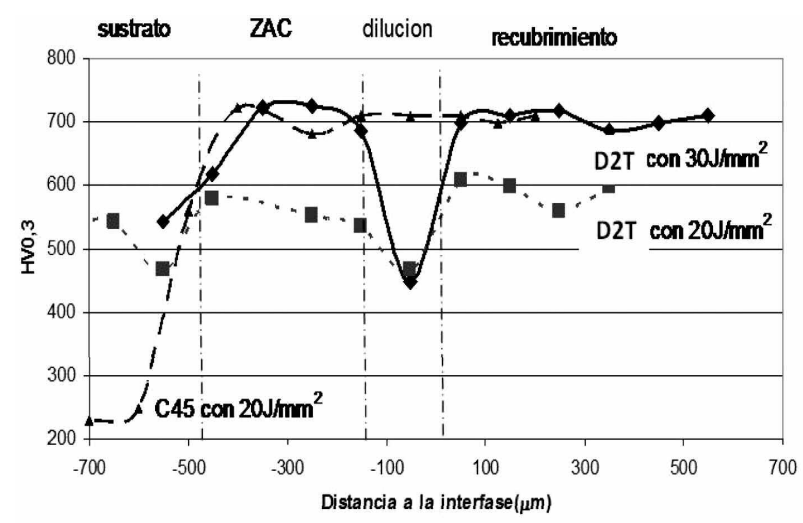

Figura 8. Variación de la microdureza a lo largo de tres recubrimientos con las mismas condiciones de procesado y diferente substrato base.

Figure 8. The variation of microhardness across three clad layers with the same process conditions and different substrate.

primera de ellas, con $30 \mathrm{~J} / \mathrm{mm}^{2}$ sobre acero para herramientas tratado y, la segunda, con $20 \mathrm{~J} / \mathrm{mm}^{2}$ sobre acero para herramientas y un acero al carbono C45 como referencia para comparar el efecto de la austenita retenida.

Los resultados muestran que una energía elevada $\left(30 \mathrm{~J} / \mathrm{mm}^{2}\right)$ consigue transformar el sustrato afectado por el calor e incrementar su dureza hasta $700 \mathrm{HV}$, que es la máxima dureza alcanzable para el acero D2 en estado de temple (D2T), mejorando el rendimiento del tratamiento previo. Además, con un enfriamiento más lento, se reduce la extensión y cantidad de austenita retenida en el cordón y en el sustrato afectado.

Sin embargo, el control de energía aportada no consigue impedir la formación de una banda de austenita retenida en la zona de dilución, donde se produce el máximo gradiente de solidificación. Si se compara con la deposición sobre acero C45 se comprueba que. Incluso, con el menor nivel de energía, no aparece este efecto en el cordón de acero H13, por lo que la cantidad de austenita retenida se debe al acero del sustrato y no al polvo empleado.

\section{CONCLUSIONES}

- La técnica de recubrimiento por láser permite la reparación de herramientas y útiles de acero D2 con mejores resultados que ninguna otra técnica convencional ya que consigue una baja afectación térmica, ausencia de defectos y una dureza equivalente a la del componente tratado térmicamente.

- La optimización de los parámetros, gracias al diseño de experimentos, juega un papel muy importante para comprender la influencia de cada parámetro sobre la microestructura formada.

- La formación de austenita retenida es un efecto perjudicial, debido al ciclo térmico impuesto por el procesado láser y puede disminuirse mediante el control de los parámetros del proceso. Sin embargo, para el par de materiales D2-H13, aunque se consigue inhibir su formación en el cordón, no se puede evitar su formación en la intercara.

- Para recubrir superficies extensas, es beneficioso elegir parámetros para el primer cordón que suministren una energía $(\mathrm{P} / \mathrm{VD})$ superior a $27 \mathrm{~J} / \mathrm{mm}^{2}$ con una velocidad de avance baja ya que el substrato está frío y se genera gran cantidad de austenita retenida. En el segundo y sucesivos cordones se puede disminuir hasta los $22 \mathrm{~J} / \mathrm{mm}^{2}$ a través de un incremento de la velocidad, manteniendo la potencia constante de manera que se minimice la distorsión de la pieza y se aumente la productividad.

\section{Agradecimientos}

Los autores agradecen al Ministerio de Educación y Ciencia por la financiación de esta investigación a través del proyecto DPI2005-09244-C04-04 enmarcado 
en el Programa de Producción Industrial del Plan Nacional y a su continuidad con el proyecto MAT200806882-C04-04/MAT del Ministerio de Ciencia e Innovación.

\section{REFERENCIAS}

[1] S. Wang, J. Chen y L. Xue, Surf. Coat. Tech. 200 (2006) 3.446-3.458.

[2] J. Grum y J.M. Slabe, Appl. Surf. Sci. 208-209 (2003) 424-431.

[3] E. Capello, D. Colombo y B. Previtali, J. Mater. Process. Tech. 164-165 (2005) 990-1000.

[4] M. Vedani, J. Mater. Sci. 39 (2004) 241-249.

[5] M. Vedani, B. Previtali, G.M. Vimercati, A. Sanvito y G. Somaschini, Surf. Coat. Tech. 201 (2007) 4.518-4.525.

[6] A. García-Beltrán, L.F. Marín y J.L. Ocaña, Rev. Metal. Madrid 43 (2007) 284-293.

[7] I. Fernández-Pariente y F.J. Belzunce-Varela, Rev. Metal. Madrid, 42 (2006) 279-286.
[8] W. Steen. Laser Material Processing. Ed. Springer. $3^{\text {rd }}$ ed. 2003.

[9] H.J. Niu y I.T.H. Chang, Metall. Mater. Trans. A, 31A (2000) 2615-2625.

[10] R. Colaço y R. Vilar, Scripta Mater. 36 (1997) 199-205.

[11] R. Colaço y R. Vilar, Scripta Mater. 38 (1998) 107-113.

[12] R. Colaço y R. Vilar, J. Mater. Sci. Lett. 17 (1998) 563-567.

[13] S. Thompson, Handbook of Mold, Tool and Die Repair Welding. Woodhead Publishing. 1999.

[14] U. Oliveira, V. Ocelík y J. De Hosson, Surf. Coat. Tech. 197 (2005) 127-136.

[15] J. Kim y Y. Peng, J. Mater. Process. Tech. 104 (2000) 284-293.

[16] R. Colaço, y C. Pina, R. Vilar, Scripta Mater. 41 (1999) 715-721.

[17] M. Bonek y L.A. Dobrzanski, J. Mater. Process. Tech. 175 (2006) 45-54.

[18] K.A. Chiang y Y.C. Chen, Mater. Lett. 59 (2005) 1.919-1.923. 